\title{
Scaring and encouraging news on diagnosis, spreading, and therapy of resistant organisms: the ECCMID 2017 in Vienna
}

\author{
Johannes R. Bogner ${ }^{1}$
}

Published online: 8 May 2017

(C) Springer-Verlag Berlin Heidelberg 2017

The European Congress of Clinical Microbiology and Infectious Diseases (ECCMID) is the annual meeting of the European Society of Clinical Microbiology and Infectious Diseases (ESCMID). This year it took place from 22 to 25 April 2017 in Vienna, Austria. Meanwhile, ECCMID is referred to as the world's largest congress combining the fields of infectious diseases and clinical microbiology. Researchers presented more than 3000 regular and late-breaking abstracts with the latest findings and recommendations, which are set to help improve diagnosis, prevention and treatment of infection-related diseases. The congress offered almost 200 sessions, including keynote lectures, symposia, oral sessions, and other interactive formats. ECCMID hosted almost 12,000 participants from more than 100 countries. Some of the highlights may be briefly referred to here as my personal selection:

One of the late breakers seems to correspond to last year's big news [2]: colistin resistance. This year a new diagnostic method was presented by Dr. Larrouy-Maumus and colleagues (Abstract OS0558). The researchers tested 134 different colonies of bacteria using a mass spectrometer. Mass spectrometers are found in testing labs at most hospitals where they are used to analyse various different molecules. They found that it was possible to distinguish not only between bacteria with and without colistin-resistance but also to test which bacteria have the more dangerous plasmid-encoded resistance. They say the test can be carried out in around $15 \mathrm{~min}$ and would cost less than one

Johannes R. Bogner

Johannes.Bogner@med.uni-muenchen.de

Sektion Klinische Infektiologie Klinik und Poliklinik IV, Klinikum der Universität München, Pettenkoferstr. 8a, 80336 Munich, Germany
US dollar per sample. Dr. Larrouy-Maumus explained: "The exciting thing about this technique is that it relies on technology that is already available in most hospitals. This means that it could be rolled out quickly and cheaply and that it might potentially have a rapid impact on tackling drug-resistance". The test could also be useful for screening in veterinary samples, where levels of colistin-resistance are known to be high.

The great international concern about the spreading of resistant organisms in animals was the topic of one session on Monday 24th. Data on difficult-to-treat infections in animals were presented. Especially the risk of transmission to humans and the possibility of a transfer of antimicrobial resistance to opportunistic pathogens present in the human commensal microbiota were presented. The session also highlighted the importance of antimicrobial stewardship in the veterinary sector and the challenges that veterinarians are facing to implement the general principles of prudent antimicrobial use in livestock and companion animals (SY\#572). Luca Guardabassi pointed at a high variability of antibiotic use, e.g. in pig farming with aminopenicillins representing 20-40 percent of antibiotics used while oral bioavailability is only in the range of $28-33 \%$ in pigs. One tool to regulate the antibiotic consumption is the benchmarking of antimicrobial use per herd and veterinarian. Other measures suggested to control the antimicrobial use are legal interventions and antimicrobial stewardship in the veterinary setting $[6,7]$.

The effect either combination therapy versus monotherapy for the treatment of blood stream infections due to ESBL enterobacteria (ESBL-E) on 30-day mortality was studied in a project called increment. The results of this multicentre retrospective cohort analysis at 26 centers in ten countries over ten years were presented by Zaira Palacios Baena. Appropriate initial therapy (78\%) was compared to 
inappropriate therapy (22\%) and stratification was according to the initial mortality score. As expected, mortality was lower in those who received appropriate therapy but among those receiving appropriate therapy, there was no difference in mortality between combination therapy and monotherapy ( 35 vs. $41 \%, p=0.28$ ). However, taking into account the predicted mortality at baseline, combination therapy was associated with lower mortality than was monotherapy in the high-mortality-score stratum (EP0429 Zaira Palacios Baena and Belén Gutiérrez-Gutiérrez et al. Lancet ID 2017 [8]).

Purvesh Khatri presented fascinating data on a new way in devising diagnostic tests for travellers to diagnose malaria. Analysing a patient's immune response not looking at antibodies but rather on mRNA patterns could be key to quickly and accurately diagnosing malaria. Gene expression triggered by an infection can be measured by the amount of each unique type of mRNA found in a blood sample. Data were combined from already available 40 previous studies, bringing together more than 3000 blood samples from patients with various infectious diseases. This included some from patients who were known to have malaria, some from patients with other common tropical diseases such as dengue, typhoid, or leishmaniasis, and some from healthy volunteers. The researchers were able to study the activity of more than 6000 different genes. They used a computer to trawl through 2100 blood samples and look for patterns of gene expression. They found that a group of seven genes showed a different pattern of expression in patients with malaria, compared with healthy people and patients with other travel-associated infectious diseases (OS174-let's diagnose parasites).

Showing their results of the diagnostic performance of tissue sonication culture versus sonication multiplex PCR from samples of 78 infected prosthetic joint infections Nora Renz and Andrej Trampuz from Berlin concluded that the performance of PCR is comparable to tissue culture while it is much more rapid and has the advantage of an automated process. However, the primer setup needs to be augmented to include also Propionibacteria, additional species of Streptococci and coagulase-negative Staphylococci (OS0122). Infection has included the topic earlier [3, 4].

Results on Hepatitis E in attendants of a STD clinic in Brussels were presented by Nicolas Dauby (OS0551). The rationale for this investigation comes from the fact that pathogens known to be transmitted by the faecal-oral route are more commonly diagnosed in men who have sex with men $(\mathrm{msm})$ than in other patient groups. 799 persons attending the clinic between June 2014 and January 2016 who received an HIV test were also tested serologically for Hepatitis E. $31 \%$ were msm. There was no significant difference in seroprevalence between $\mathrm{msm}$ and non-msm-participants. HEV overall prevalence was $9 \%$ and that does not differ from other seroprevalence data from Belgium. The only factor associated with higher seroprevalence was higher age. Only two persons were found to have positive IgM for HEV. INFECTION has reported on the growing awareness of HEV not only in special risk populations but also in the area of transfusion services [10].

Another presentation in the session on emerging viruses reported on characteristics of a new arbovirus Mayaro virus (MAYV; OS0552). Remý Mutricy was the presenter of retrospective data from French Guiana 2003-2016. MAYV IgM testing was performed in all samples submitted for dengue and chikungunya-testing. Nine patients with high and medium clinical likelihood of acute disease were included in further analysis. Fever, headache and arthralgia were the most common symptoms. Laboratory features were lymphopenia and thrombocytopenia. No severe or fatal courses were observed. Thus, Mayaro virus infection in symptomatic patients is rated as not dangerous. However, differential diagnosis of dengue fever may be important because dengue may proceed to fatal disease. Differential diagnosis of arthralgias with travel-associated alphaviruses fevers has been presented in a review by Suhrbier [9].

OS0558 Zika virus and its characteristics in clinical and laboratory differentiations from dengue and chikungunya infection were investigated by Milan Trojanek and colleagues from Prague. They pointed out that in Zika virus infection fever and arthralgia was less frequent while conjunctivitis was observed in 57\% (versus 13\% in dengue and $30 \%$ in chikungunya). Looking at routine laboratory parameters, the average platelet count and liver function tests were lower in Zika virus infection [1,5].

Again, the spectrum of diagnostic and clinical papers at this conference was not only overwhelming but also innovative and representing positive development in many areas. Many participants are already booking and looking forward to the next ECCMID 2018 in Madrid (April 21-24, 2018). Meanwhile, let us have a look at presentations that will appear in infectious disease journals, including INFECTION.

\section{Compliance with ethical standards}

Conflict of interest The author declares that he has no conflict of interest.

\section{References}

1. Atif M, Azeem M, Sarwar MR, et al. Zika virus disease: a current review of the literature. Infection. 2016;44:695-705. 
2. Bogner JR. Blue sky and some shadows: new antibiotics and new superbugs: the ECCMID 2016 in Amsterdam. Infection. 2016;44:271-2.

3. Borde JP, Hacker GA, Guschl S, et al. Diagnosis of prosthetic joint infections using UMD-Universal Kit and the automated multiplex-PCR Unyvero i60 ITI((R)) cartridge system: a pilot study. Infection. 2015;43:551-60.

4. De Angelis G, Mutters NT, Minkley L, et al. Prosthetic joint infections in the elderly. Infection. 2015;43:629-37.

5. Duijster JW, Goorhuis A, Van Genderen PJ, et al. Zika virus infection in 18 travellers returning from Surinam and the Dominican Republic, the Netherlands, November 2015-March 2016. Infection. 2016;44:797-802.

6. Guardabassi L, Hedberg S, Jessen LR, et al. Optimization and evaluation of Flexicult(R) Vet for detection, identification and antimicrobial susceptibility testing of bacterial uropathogens in small animal veterinary practice. Acta Vet Scand. 2015;57:72.

7. Guardabassi L, Prescott JF. Antimicrobial stewardship in small animal veterinary practice: from theory to practice. Vet Clin North Am Small Anim Pract. 2015;45:361-76.

8. Gutiérrez-Gutiérrez B, Slamanca E, Decueto M (2017) Effect of appropriate combination therapy on mortality of patients with bloodstream infections due to carbapenemase-producing Enterobacteriaceae (INCREMENT): a retrospective cohort study. Lancet Infectious Diseases ePub ahead of print.

9. Suhrbier A, Jaffar-Bandjee MC, Gasque P. Arthritogenic alphaviruses-an overview. Nat Rev Rheumatol. 2012;8:420-9.

10. Yazbek S, Kreidieh K, Ramia S. Hepatitis E virus in the countries of the Middle East and North Africa region: an awareness of an infectious threat to blood safety. Infection. 2016;44:11-22. 